\title{
Coordinated management of low voltage power networks with photovoltaic energy sources
}

\author{
Brenda Rojas, Mónica Alonso, Hortensia Amarís, Lorena González \\ Universidad Carlos III deMadrid, Avda de la Universidad nº30, Leganés, 28911, Spain
}

\begin{abstract}
Over the last decades, active power networks have reached great attention due to the incorporation of distributed energy resources into low voltage power systems. In this paper, a decentralized energy management strategy is proposed as an efficient way to minimize both active power losses and voltage profile deviation of an distribution power network with photovoltaic solar farms, and also at the same time, aims to improve the reliability and the security of supply. The coordinated energy management concept relies on a two-step optimization approach based on genetic algorithms (GA) and MINLP, in which a multi-objective function is used which takes into account reliability and operational technical constraints in its formulation. The suitability of the proposed methodology is tested on an existing low voltage power system, in which two aspects are considered: firstly, determining the optimal allocation of PV units and secondly, establishing the optimal reschedule of the active power of the generation units participating in the energy mix and minimizing both the real power losses and voltage deviation of the entire power system.
\end{abstract}

Keywords: Renewable energy, PV plants, genetic algorithms, smart grids

\section{Introduction}

Solar PV market has slightly increased during 2014 from 37.6 GW in 2013 to 38.7 GW [1] and its future expectations are very promising because of several factors. PV energy has reduced its unit costs which happened thanks to continuous technological progress, higher production efficiency and its widespread implementation, among other reasons. The trend of decreasing unit cost will also continue in the future as the technology improves. Thus, $\mathrm{PV}$ is predicted to achieve grid parity (competitiveness with electricity grid retail prices) before 2020 in many regions [2].

As solar panels can be easily incorporated into the architecture of a building and because of the fact that most electricity consumption is generated within the urban environment, Building Integrated PV (BIPV) has become one of the most promising applications of PV systems. Meanwhile, there are still large industrial PV systems producing enormous quantities of electricity at a single point and which are environmentally friendly. These types of electricity generation plants can range from many hundreds of kilowatts to several megawatts.

Distribution Power network management with the presence of PV should evolve from an uncoordinated scheme, in which each DER unit is connected to the network and which works locally, to a coordinated power management throughout the whole power network in which every DER unit is controlled by a decentralized energy management controller, as shown in Fig. 1. A new power network management strategy has to be applied in order to efficiently increase the use of DER units in power networks management, and at the same time, to keep reliability of the power systems.

The decentralized energy management strategy must be in charge of optimizing the operation of the whole network by increasing the reliability and the security of supply, and at the same time, by minimizing real power losses and voltage deviation.

Manuscript received May 24, 2016; revised October 14, 2016.

Corresponding author. Tel.: +34-916-24-8853; E-mail address: brojas@ing.uc3m.es.

doi: $10.12720 /$ sgce $5.4 .213-220$ 


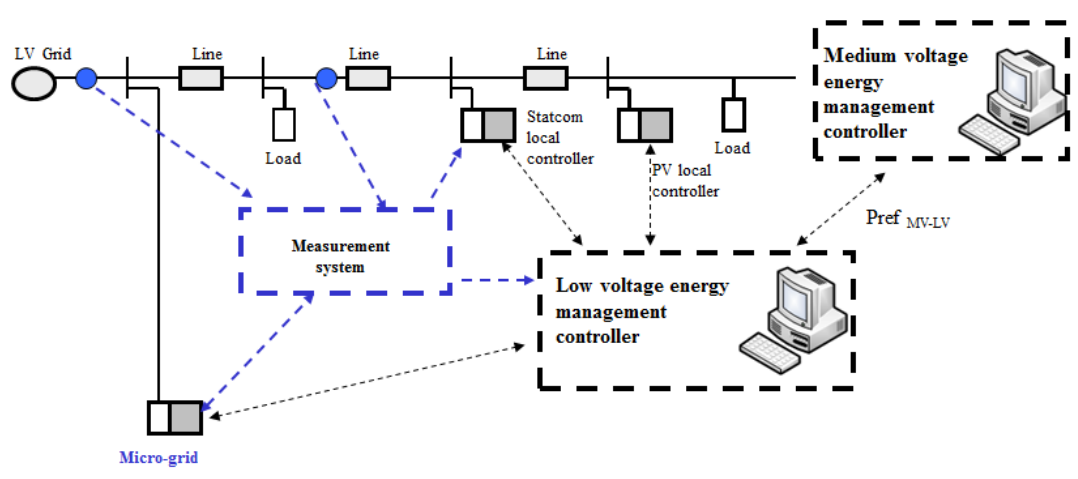

Fig. 1. Coordinated power management strategy

The traditionally used optimization algorithms [3]-[5], are no longer capable of managing non-convex systems like the proposed power system and they do not offer the availability of working with multiobjective targets. The use of a multi-objective optimization algorithm stands out as the only suitable way to design and to optimally locate DER units in power networks and, at the same time, to consider a wide range of objective functions such as: reducing active power losses, voltage deviation and improving DER penetration. Compared with single objective optimization techniques, the multi-objective ones offer advantages because they are able to provide a solution containing different trade-offs among individual objectives, thus enabling the Power System Operator to select the best final solution [3]-[9].

The objective of the paper is to develop a decentralized coordinated power management strategy between Medium Voltage (MV) distribution power system and the Low Voltage (LV) distribution network in which some PV plants are optimally located in the lower voltage level. The optimal coordination among PV sources and the MV relies on an optimization algorithm based on a MINLP that aims, in the multi-objective formulation, to minimize real power losses and voltage deviation of the whole power system, taking into account the active generation reference set point supplied by the MV network.

The suitability of the proposed strategy has been demonstrated on an existing Low Voltage distribution power system and it has been shown that the coordinated scheduling of PV plants will allow reduction of real power, at the same time, voltage deviation.

Nomenclature

\begin{tabular}{cl}
\hline$\alpha$ & Weight factor respect to losses \\
$\beta$ & Weight factor respect to deviation \\
$\theta$ & Angle between active and reactive power \\
$B_{i k}$ & Susceptance \\
DER & Distributed Energy Resources \\
DSO & Distribution System Operator \\
$\cos \theta_{i k}$ & Power factor on node $\mathrm{i}$ \\
$G i k$ & Conductance \\
$I$ Branch,j & Current on line $\mathrm{j}$ \\
MINLP & Mixed Integer Nonlinear Programming \\
$N$ & Set of buses of the network \\
$P d i$ & Active power demand at node i \\
$P g i$ & Active power generated at node i \\
$P_{P V i}$ & Active power delivered by each PV \\
$P_{\text {ref } M V-L V}$ & Active power set-point received from MV network at the PV-LV connection point \\
$\mathrm{Q} d i$ & Reactive power demand at node i \\
$\mathrm{Q} g i$ & Reactive power generated at node i \\
$U i$ & Voltage magnitude on node i \\
\hline
\end{tabular}




\section{Aims and Objectives of the Work}

The aim of this paper is to develop a coordinated optimization algorithm for renewable energy sources in LV distribution network, considering the active power set-point received from the MV network (upper level) at the connection point.

The optimal coordinated energy management strategy proposed in this paper is composed of two parts:

- First: The optimal location of low voltage PV sources is found taking into account technical operation constraints. The output of the optimization process is the optimal location point at the LV network and the optimal sizing of each PV generation units.

- Second: The optimal scheduling of each PV source for the 24 hour-ahead period is performed considering both the technical operation constraints and the active power reference set point received from the MV network

The objective is to optimize the generation delivered by involved PV plants, minimizing both active power losses and voltage deviations given by the difference between maximum desired voltage and the voltage values in each node of the MV / LV grid [10].

What is being achieved with this work is a coordinated management of low voltage networks using photovoltaic power plants, taking into account technical and operational concerns. To do so, it was developed an optimization algorithm with the structure described in III.

\section{Procedure Followed}

\subsection{Multi-objective optimization algorithm}

Traditionally, optimization problems related to multiple objectives have been solved by means of linear programming in which one of the objectives is optimized and the others are included in the restrictions. This procedure generates disadvantages such as:

- Representation of the objectives by means of the restrictions in linear programming can lead to unfeasible situations.

- If the optimization is applied in a large system it is difficult to determine which restriction is generating the unfeasibility.

- There is not a clear criterion for choosing the suitable objective function and in many cases the fulfilment of one single objective can come into conflict with others.

Non commensurable and conflicting multi-objective functions must be faced by a set of optimal solutions instead of by using just an optimal solution. This set of solutions is known as Pareto-optimal solution and represents the best trade-off among all the individual objectives. Once a Pareto optimal solution has been found, it cannot be improved with respect to any objective without worsening, at least, another objective.

Multi-objective algorithms stand out as a procedure to solve these problems in which the optimal solution is obtained by a set of efficient solutions.

$$
\min F(x)=\alpha f_{1}(x)+\beta f_{2}(x)
$$

where:

$\left\{f_{1}, f_{2}\right\}$ set of the objectives to satisfy,

$f_{l}(x)$ minimize active power losses,

$f_{2}(x)$ minimize voltage deviation.

Using different weights for $\alpha$ and $\beta$ allows several solutions to be obtained. In this paper, two main objectives have been considered: minimizing real power losses and voltage deviation.

\subsection{Optimization problem statement}

The main objective of the optimization algorithm is to minimize both active power losses (3) and voltage deviation (4). 


$$
\begin{aligned}
& f_{1}(x)=\text { losses }=\sum_{i} \sum_{j} G_{i j}\left(U_{i}^{2}+U_{j}^{2}-2 U_{i} U_{j} \cos \left(\theta_{i}-\theta_{j}\right)\right) \\
& f_{2}(x)=\text { deviation }=\operatorname{sqrt} \sqrt{\left[\left(U_{\text {imax }}-U_{i}\right)^{2} / N\right]}
\end{aligned}
$$

To get success with this implementation and achieve the main goal as well, it is necessary to put some restrictions on the grid developments, which are presented, as follows:

- Load flow equations (5)-(8)

- Voltage limits at the nodes of the network (9)

- Generator limits (10)-(11), given by the PQ curve on PV generator

- Lines and transformers overloads (12)-(13)

- Solar Resource Availability (14)

- PV generation deviations from the MV active generation set point $\left(P_{\text {refMV-LV }}\right)(15)$

$$
\begin{aligned}
& P_{g i}-P_{d i}-P_{i}(U, \theta)=0 \\
& Q_{g i}-Q_{d i}-Q_{i}(U, \theta)=0 \\
& P_{i}(U, \theta)=U_{i} \sum_{k=1}^{N} U_{k}\left(G_{i k} \cos \theta_{i k}+B_{i k} \sin \theta_{i k}\right) \\
& Q_{i}(U, \theta)=U_{i} \sum_{k=1}^{N} U_{k}\left(G_{i k} \sin \theta_{i k}-B_{i k} \cos \theta_{i k}\right) \\
& U_{i, \text { min }} \leq U_{i} \leq U_{i, \text { max }} \\
& P_{g i, \text { min }} \leq P_{g i} \leq P_{g i, \text { max }} \\
& Q_{g i, m i n} \leq Q_{g i} \leq Q_{g i, \text { max }} \\
& I_{\text {brach } j} \leq I_{\text {máx,branch } j} \\
& P_{\text {transformer }} \leq P_{\text {máx,transformer }} \\
& P_{P V i, h} \leq P_{\text {máx }, h} \\
& \sum_{i} P_{P V_{i}} \leq P_{\text {refmV-LV }}
\end{aligned}
$$

$P_{r e f M V-L V}$ is referred as the active power set-point received from MV network (upper level) at the MVLV connection point. The MINLP algorithm proposed in this paper was developed and simulated on MATLAB environment [11]-[12].

\section{Application Results}

\subsection{Description of the low voltage network}

In the next section, the proposed coordinated management strategy based on multi-objective MINLP is applied upon an existing LV distribution power network (380V), consisting of five main feeders (162 nodes) which feeds to 56 residential consumers, with a total contracted capacity of $1.87 \mathrm{MW}$.

\subsection{First step: Optimal sitting and sizing of PV plants}

The first step in the optimization of the power system is to accommodate several PV plants. Three PV plants with a total capacity of $100 \mathrm{~kW}$ must be optimally connected to the LV power network. 
Optimal evacuation point and proper sizing of PV plants are found by using an optimization algorithm in which a multi-objective GA strategy is applied in order to:

- Minimize real losses, $f_{l}(x)$,

- Minimize voltage deviation, $f_{2}(x)$,

Thereby, the multi-objective function could be expressed as (2).

According to Spanish national legislation, every utility is obliged to maintain the voltage supply within an admissible margin of $10 \%$ around nominal voltage [8]. Consequently, this restriction is considered to be a constraint in the optimization problem.

The results of the multi-objective process are shown in Table 1. The optimal evacuation point of a solar PV plant should be located at bus \#127 with a size of $60 \mathrm{~kW}$ on feeder 1, a second solar PV plant has to be located at bus \#217 with a rate of $35 \mathrm{~kW}$ on feeder 2 and finally, a third solar PV plant is to be located at bus \#419 with a rate of $5 \mathrm{~kW}$ on feeder 4 .

Table 1. Optimal allocation and size

\begin{tabular}{cccccc}
\hline \multicolumn{2}{c}{$\mathrm{PV}_{1}$} & \multicolumn{2}{c}{$\mathrm{PV}_{2}$} & \multicolumn{2}{c}{$\mathrm{PV}_{3}$} \\
\hline Loc $_{\mathrm{PV} 1}$ & Rate $_{\mathrm{PV} 1}$ & Loc $_{\mathrm{PV} 2}$ & Rate $_{\mathrm{PV} 2}$ & Loc $_{\mathrm{PV} 3}$ & Rate $_{\mathrm{PV} 3}$ \\
127 & {$[\mathrm{~kW}]$} & & {$[\mathrm{kW}]$} & & {$[\mathrm{kW}]$} \\
& 60 & 217 & 35 & 419 & 5
\end{tabular}

Table 2 shows how the optimal evacuation point of PV sources can reduce power losses up to $20 \%$, compared to the original power network.

Table 2. Power losses

\begin{tabular}{ccc}
\hline $\begin{array}{c}\text { Original network } \\
\text { (without PV } \\
\text { sources) }\end{array}$ & $\begin{array}{c}\text { Optimal network } \\
\text { (PV sources } \\
\text { optimally located) }\end{array}$ & $\begin{array}{c}\text { Power losses } \\
\text { reduction }\end{array}$ \\
\hline $75 \mathrm{~kW}$ & $60 \mathrm{~kW}$ & $20 \%$ \\
\hline
\end{tabular}

\subsection{Second step: Optimal day-ahead scheduling}

Once the evacuation point of PV plants has been optimally located and sized in the power network according to Table 1, an optimal coordinated scheduling of real power generation from conventional and solar PV plants is performed.

The second step of the optimization process deals with minimization of both real power losses and voltage deviation of the whole power systems for the next 24 hour day-ahead, taking into account the MV active reference set point received from the MV upper level. The inputs of this process are:

- The 24 hour Day-Ahead load forecast provided by the Distribution System Operator (DSO).

- The 24 hour Day-Ahead solar generation forecast as detailed in [13].

- The optimal power network topology where PV plants have been optimally located and sized.

- The MV-LV active reference set point.

With all these inputs, MINLP accommodates real power generation of all generation units in an optimal and decentralized way according to (2).

Several optimization strategies have been developed and summarize in Table 3.

Table 3. Optimization strategies

\begin{tabular}{lccc}
\hline & PV & $\alpha$ & $\beta$ \\
\hline O.E. 1 & optimal & 0 & 1 \\
O.E. 2 & optimal & 0.5 & 0.5 \\
O.E. 3 & optimal & 1 & 0 \\
\hline
\end{tabular}

For each one of the three optimization strategies, two different scenarios are considered: typical winter day and summer day. 


\subsection{Winter and summer day scenarios}

A typical winter and summer day scenario is summarized in Appendix. The hourly load demand and the 24 hour Day-Ahead solar generation forecast for the winter day are shown in Fig. 5 (a) and (b) respectively. Knowing the 24 hour day ahead reference set point $\left(P_{\text {ref } M V-L V}\right)$ received from the MV level, the different optimization strategies are compared in terms of reduction of power losses (in percentage) and voltage deviation in p. u. Fig. 2 shows the reduction of power losses (in percentage) for each one of the optimization strategies detailed in Table 3. It could be observed that only those optimization strategies which include the minimization of active power losses in its formulation are able to reduce power losses during the whole day which corresponds to O.E.2 and O.E.3.

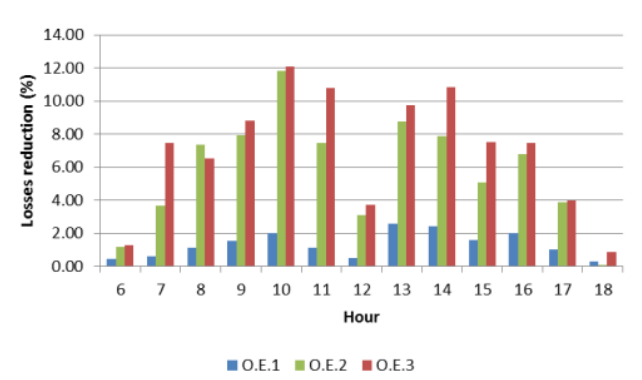

(a)

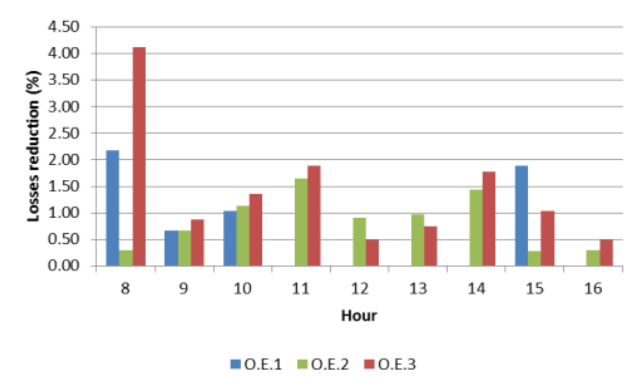

(b)

Fig. 2. Losses reduction for winter (a) and summer scenario (b).

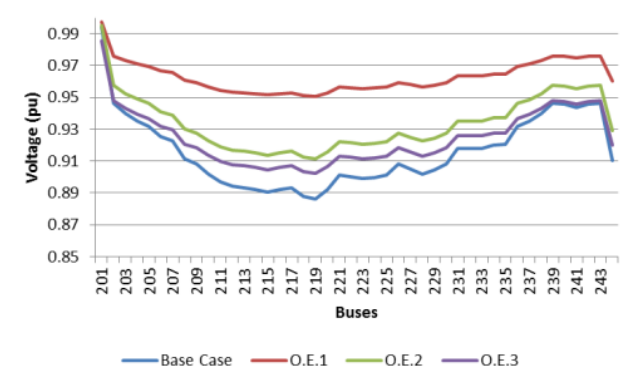

(a)

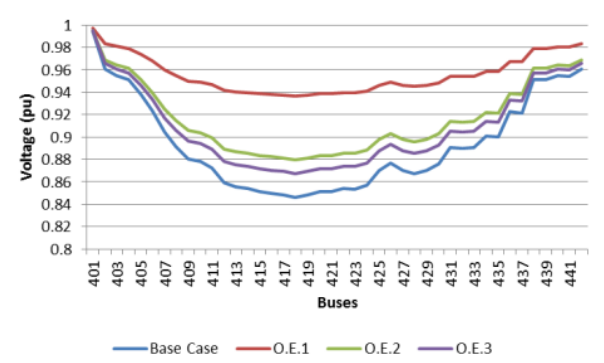

(b)

Fig. 3. Voltage profile for peak hour and winter (a) and summer (b) scenario.

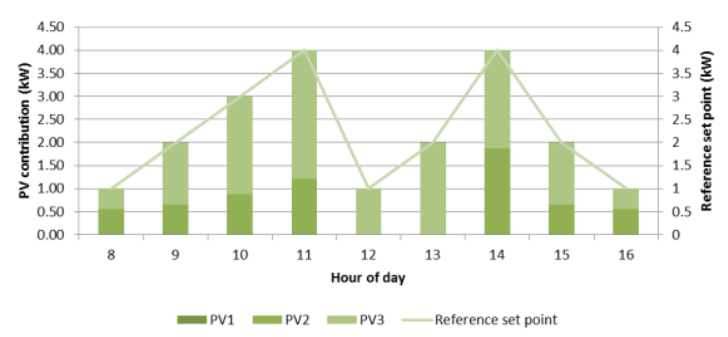

(a)

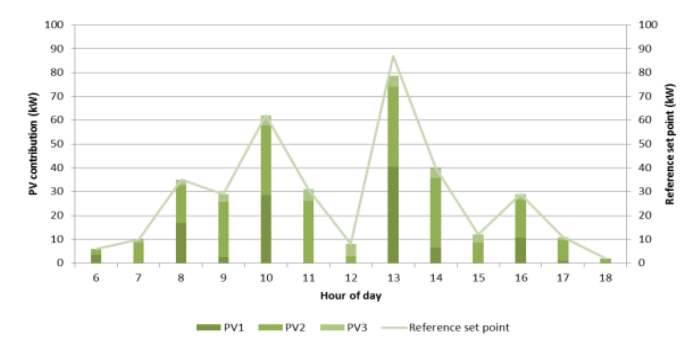

(b)

Fig. 4. PV generation for O.E.2 winter (a) and summer (b) scenario.

Fig. 3 represents the voltage profile for the peak demand hour in main feeders with presence of PV. It can be seen that incorporation of PV plants improves the voltage profile, in terms of voltage deviation for all the optimization strategies. In this case, O.E.1 is the strategy that allows voltage profile to be flatter.

Fig. 4 shows the hourly contribution of each PV source for complying with the active power reference set point received from the MV level when O.E. 2 is considered. It can be noted that PV2 and PV3 are the most suitable units to fulfil the generation of the commanded reference set point, $P_{\text {ref } M V-L V}$, received from 
the upper level.

From previous results it can be concluded that the application of multi-objective optimization strategies improves the technical operation of the distribution network minimizing both active power losses and voltage profile deviation.

At the same time, the multi-objective optimization formulation based on MINLP proposed in this paper is very effective to coordinate in a decentralized way the whole operation of MV and LV distribution power networks when PV units are included.

\section{Conclusions and Future Approach}

This paper presents a decentralized coordinated management strategy in active networks where conventional and distribution energy sources such as photovoltaic power plants are operating in a coordinated and optimum way. The Day-ahead unit commitment scheduling is performed by using the proposed optimization strategy, in which the power production of every generation unit (conventional and renewable) is optimally planned according to the 24 hour Day-Ahead forecast of the power demand supplied by the DSO. The method has been applied to an existing low voltage distribution power network in which photovoltaic power plants are optimally located using GA. Results show the capability of the proposed optimization strategy based on MINLP to maintain the reliability and safety of the supply of the active power network.

Future approach has driven by a centralized medium and low voltage coordinated management that will be developed to deal with the optimal day-ahead unit commitment of a whole distribution power system, from medium to low voltage power system.

\section{Appendix}

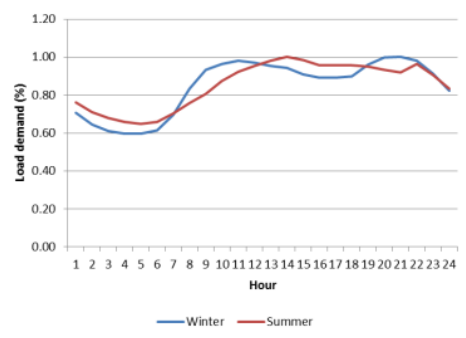

(a)

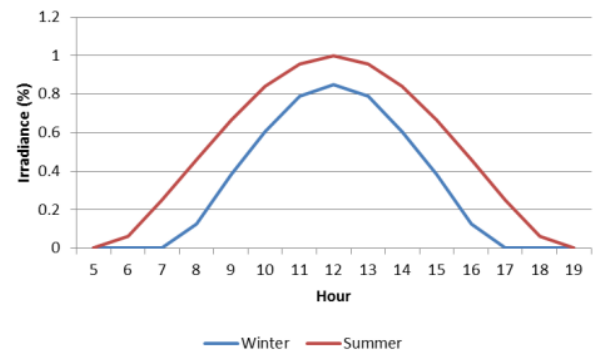

(b)

Fig. 5. 24-hour day-ahead power demand forecast (a) and Solar forecast of PV plants (b)

\section{Acknowledgement}

This work has been partly funded by the European Union seventh framework program FP7SMARTCITIES-2013 under grant agreement 608860 IDE4L - Ideal grid for all.

\section{References}

[1] A Snapshot of Global PV (1992-2014). Report IEA PVPS T1-26:2015.

[2] I. E. A. Technology Roadmap Solar PV energy. 2010.

[3] Zhang W, Li F, Tolbert LM. Review of reactive power planning: objectives, constraints and algorithms. IEEE Trans. on Power Systems, 2007; 22(4):2177-2186.

[4] Fuller JF, Fuchs EF, Roesler KJ. Influence of harmonics on power distribution system protection. IEEE Trans. Power Delivery, 1998; 3:549-557.

[5] Dommel HW, Tinney WF. Optimal power flow solutions. IEEE Trans. on Power Apparatus and Systems, 1968; 87:1866-1876.

[6] Alonso M, Amaris H, Alvarez-Ortega C. A multiobjective approach for reactive power planning in networks with wind power generation. Renewable Energy, 2012; 37:180-191. 
[7] Alonso M, Amaris H, Chindris M. A novel genetic algorithm for the coordinated optimal reactive power dispatching power systems with wind farms and FACTS. International Review of Electrical Engineering, 2011; 6(2):826-835.

[8] Amaris H, Alonso M. Coordinated reactive power management in power networks with wind turbines and FACTS devices. Energy Conversion and Management, 2011; 52:2575-2586.

[9] Brini S, Ben Aribia H, Abdallah H, Ouali A. Multiobjective optimization of the active/reactive/environmental dispatch of an electrical network. International Review of Electrical Engineering, 2007; 2:301-309.

[10] Singh D, Singh D, Verma KS. Multiobjective optimization for DG planning with load models. IEEE Transactions on Power Systems, 2009; 24:427-436.

[11] MATLAB and Statistics Toolbox Release 2013b, The MathWorks, Inc., Natick, Massachusetts, United States.

[12] Zimmerman RD, Murillo-Sánchez CE, Thomas RJ. MATPOWER: Steady-State operations, planning and analysis tools for power systems research and education. IEEE Transactions on Power Systems, 2011; 26(1):12-19.

[13] Koca A, Oztop HF, Varol Y. Estimation of solar radiation using artificial neural networks with different input parameters for Mediterranean region of Anatolia in Turkey. Expert Systems with Applications, 2011; 38(7):8756-8762. 